\title{
MODIFICATION OF MODAL PROPERTIES OF A SYSTEM WITH NON-PROPORTIONAL DAMPING DUE TO VIBRATION ABSORBERS AND SUB-SOIL INTERACTION
}

\author{
Jiří Náprstek ${ }^{1}$ and Stanislav Hračov ${ }^{1}$ \\ ${ }^{1}$ Institute of Theoretical and Applied Mechanics AS CR, v.v.i. \\ Prosecká 76, CZ-19000, Prague, Czech Republic \\ e-mail: $\{$ naprstek,hracov\} @itam.cas.cz
}

Keywords: Non-proportional damping, perturbation method, modal properties.

\begin{abstract}
The paper deals with a complex linear dynamic system with proportional damping, which is completed by various elements making the final system non-proportionally damped. Such effects emerge due to vibration absorbers, soil-structure interaction and other components and details. Influence of subsequently attached elements being a source of the non-proportional damping are reflected using perturbation method on the level of the first degree of a small parameter. This parameter characterizes the damping non-proportionality as the relevant perturbation. In the role of the first step the proportionally damped system is adopted. The basic system includes such a part of individual supplements to keep the proportional damping. Eigen-functions of the basic system are then used as a certain variational basis for approximate analysis of eigen-values and eigen-modes of the original system respecting non-proportionality.

Wide numerical experiments have been carried out investigating interaction of eigen-modes between resonance domains. Applicability and shortcomings of proposed analytical-numerical procedures are commented. A few hints to improve the dynamic model fidelity and to reduce an effort of numerical evaluation are given. A couple of recommendations for engineering application of supplementary elements concerning their dynamic properties are indicated. Some open problems and perspectives of further development are outlined.
\end{abstract}




\section{INTRODUCTION}

Slender structures like TV towers, guyed masts, industrial chimneys and others are subdued to wind excitation, seismic attacks and other dynamic loading types. Therefore these structures as a rule are fitted with passive vibration absorbers of various types. Structure itself can be mostly considered as proportionally damped whether it is realized of one (e.g. mast: steel) or several materials (e.g. tower: concrete-steel-composite). However internal damping of the absorber is always significantly higher than that of the structure and so the dynamic character of the system as a whole becomes also non-proportional, see e.g. [1],[2]. Analogously dynamic interaction of the structure with the subsoil leads obviously to non-proportional damping. Some more structural details of the structure contribute to the non-proportionality as well.

In principle such a system should be investigated in a complex domain which means that the number of response components would be as twice as higher in comparison with a classical proportional approach. However the character of the system combining structure with a vibration absorber, usually enables combine the modal parameters (eigen-values and eigen-modes) of the basic "nearly proportional" system with subsequently attached strongly non-proportional supplements. Indeed such a synthesis is approximately exerting to obtain modal characteristics of the whole on a basis of modal characteristics of individual parts. This strategy enables a good insight to internal character of the structure, to optimize transparently vibration absorbers in a particular case and assess the system in a very wide extent of parameters. The reason is, that a great part of the analysis is based on analytical procedures having always more general effect than merely a numerical analysis.

To analyze the structure itself independently is inherent as it is rather geometrically large having many degrees of freedom or being modeled with continuously distributed stiffness and mass. Its effect within the system can be respected using a limited number of modal parameters only, so the transparency of the final analysis remains still obvious. On the other hand the vibration absorber is usually well examined subsystem with one or slightly more degrees of freedom well discussed independently. The same holds regarding influence of the subsoil.

Let us mention that problems of nonproportional damping has been defined and dealt in detail in classical monographs as well as in a number of papers throughout several decades, see e.g. [3], [4], [5], [6]. The dynamic response of non-proportionally damped structures is usually solved excluding the very time consuming direct method using state-space method, see e.g.[7]. The dimension of the original system is doubled and its complex eigen-modes are subsequently used for uncoupling of differential equations. The high numerical demand especially on the calculation of the complex eigen-modes has motivated many authors to define criterions for neglecting of mechanical interaction between real eigen-modes, see e.g. [8], [9], [10]. These criteria asses errors introduced when solving the problem using classical modal superposition. Another efficient approach is based on the decomposition of the modal damping matrix into the diagonal and non-diagonal parts. Then the problem is solved using an iterative method, see e.g. [11], [12]. Advantage of this strategy consists in application of matrices of the original dimension, however it doesn't provide full insight into the physics of the problem.

During last years the authors have focused on study of the complex modal properties and reductions of numerical problems of their evaluation. Adhikari [13] analyzed the sensitivity of modal properties on changes in damping, stiffness and mass of the system. Based on the assumption of small damping Woodhouse [14] and later Adhikari [15] expressed the complex eigen-modes as a combination of real undamped modes. Hračov et al. [16] has performed parametric analysis of an over-damping of individual eigen-values of double degree of freedom 
system. Garvey et al. [17] determined the relation between the real and complex parts of eigenmodes for the system with symmetrical structural matrices. The new methods of eigen-problem based on e.g. Lanczos method [18] or complex sub-space iteration method [19] have been evaluated. Main and Krenk [20] proposed an approximate eigen-problem solution of a structure with viscous dampers, being obtained as a result of interpolation between the undamped and constrained eigen-problems. Finally also the widely used perturbation theory has been applied to eigen-solution of the nonproportionally damped structures, see e.g. [21], [22], [23], [24].

In this paper the modal properties of the slender structure equipped with the absorber have been evaluated using the perturbation method of the first order. The accuracy of proposed method has been verified and their results compared with the exact solution. The recommendations and limitations for application to certain types of structures are given and discussed.

\section{THEORETICAL BACKGROUND}

Eigen vibration of a slender console shaped structure approached as a linear one dimensional system with continuously distributed mass and stiffness completed by a linear vibration absorber can be described as follows:

$$
\begin{aligned}
&\left(E J u^{\prime \prime}\right)^{\prime \prime}+\left(N u^{\prime}\right)^{\prime}+2 b \dot{u}+\mu \ddot{u}+\delta_{q}\left[C\left(u_{q}-u_{d}\right)+2 b_{d}\left(\dot{u}_{q}-\dot{u}_{d}\right)\right]=0 \\
& m \ddot{u}_{d}-C\left(u_{q}-u_{d}\right)-2 b_{d}\left(\dot{u}_{q}-\dot{u}_{d}\right)=0
\end{aligned}
$$

$E J=E J(x), N=N(x), b=b(x), \mu=\mu(x)$ - bending stiffness, static axial force, total damping (internal viscosity, aerodynamic dissipation), mass; they are characterized by piecewise continuous positive integrable functions of the length coordinate $x$;

$C, b_{d}, m$ - characteristics of the absorber; $b_{d}=\zeta_{d} \cdot \sqrt{C \cdot m}$, where $\zeta_{d}$ is the damping ratio;

$u=u(x, t), u_{q}=u\left(x_{q}, t\right), u_{d}=u_{d}(t)$ - shaft deviation, shaft deviation in point of the absorber attachment, absorber deviation;

$\delta_{q}=\operatorname{dir}\left(x-x_{q}\right)-x_{q}$ is the point of the absorber attachment;

The construction should be kinematically stable with respect to main boundary conditions in order to ensure the solution uniqueness.

To investigate modal characteristics of the system Eq. (1) the exponential time history of the response should be expected, namely:

$$
u(x, t)=v(x) \exp (\mathrm{i} \omega t), \quad u\left(x_{q}, t\right)=v_{q} \exp (\mathrm{i} \omega t), \quad u_{d}(t)=v_{d} \exp (\mathrm{i} \omega t) .
$$

Substituting expressions Eq. (2) into Eqs (1) one obtains for amplitudes:

$$
\begin{aligned}
\left(E J v^{\prime \prime}(x)\right)^{\prime \prime}+\left(N v^{\prime}(x)\right)^{\prime}+ & 2 \mathrm{i} \omega b v(x)-\mu \omega^{2} v(x)+ \\
+\delta_{q}\left[C\left(v_{q}-v_{d}\right)+2 \mathrm{i} \omega b_{d}\left(v_{q}-v_{d}\right)\right] & =0, \\
- & m \omega^{2} v_{d}-C\left(v_{q}-v_{d}\right)-2 \mathrm{i} \omega b_{d}\left(v_{q}-v_{d}\right)=0 .
\end{aligned}
$$

The definition domain of the above operator is finite. The relevant operator is symmetric and boundary conditions make the body fixed. Then the solution of the system Eq. (3) is an eigenvalue problem. In other words there exist an infinite set of eigen-values and eigen-modes which satisfies Eq. (3) together with respective boundary conditions. Let us assume, that all eigenmodes are simple and that mutual assigning of eigen-values and eigen-modes is single-valued. 
According with the previous paragraph we concentrate in the first step to solve the eigenvalue problem of the system Eq. (3) with canceled damping. The reduced operator obviously reads:

$$
\begin{gathered}
\begin{aligned}
\mathbf{L}\left\{v_{j, 0}(x)\right\}+ & \delta_{q} C\left(v_{q j, 0}-v_{d j, 0}\right)=\omega_{j, 0}^{2} \mu(x) \cdot v_{j, 0}(x), \\
& -C\left(v_{q j, 0}-v_{d j, 0}\right)=\omega_{j, 0}^{2} m \cdot v_{d j, 0},
\end{aligned} \\
\text { where: } \quad \mathbf{L}\{v(x)\} \equiv\left(E J(x) v^{\prime \prime}(x)\right)^{\prime \prime}+\left(N(x) v^{\prime}(x)\right)^{\prime},
\end{gathered}
$$

together with boundary conditions:

$$
\begin{aligned}
& \text { - top of the structure: } v_{j, 0}^{\prime \prime}(l)=0, \quad\left(E J(x) v_{j, 0}^{\prime \prime}(x)\right)^{\prime}+\left.N(x) v_{j, 0}^{\prime}(x)\right|_{x=l}=0, \\
& \text { - foot of the structure: } v_{j, 0}(0)=v_{j, 0}^{\prime}(0)=0 .
\end{aligned}
$$

Existence of the discrete spectrum of eigen-values $\omega_{j, 0}$ is guaranteed by positive definiteness (including symmetry) of the operator Eqs (4)-(7). Each eigen-value $\omega_{j, 0}$ is simple, so only one eigen-mode $\left(v_{j, 0}(x), v_{d j, 0}\right)$ is assigned to it. It can be shown that eigen-modes are orthogonal in the meaning:

$$
\begin{array}{r}
\int_{S} v_{k, 0}(x) \mathbf{L}\left\{v_{j, 0}(x)\right\} \mathrm{d} x+C \cdot\left(v_{q k, 0}-v_{d k, 0}\right)\left(v_{q j, 0}-v_{d j, 0}\right)=\left\langle\begin{array}{rl}
K_{j j}, & j=k \\
0 . & j \neq k
\end{array}\right. \\
\int_{S} \mu(x) v_{k, 0}(x) v_{j, 0}(x) \mathrm{d} x+m v_{d k, 0} v_{d j, 0}=\left\langle\begin{array}{rl}
M_{j j}, & j=k \\
0 . & j \neq k
\end{array}\right.
\end{array}
$$

Using results of lengthy reasoning which is based on a general functional form of coefficients, it can be shown, that the solution of Eq. (3) without damping can be written in a form of a convergent series in generalized coordinates $a_{i j}$ :

$$
v_{j, 0}(x)=\sum_{i=1}^{n} a_{i j} w_{i}(x), \quad v_{q, 0}=\sum_{i=1}^{n} a_{i j} w_{q i}, \quad v_{d, 0}=\sum_{i=1}^{n} a_{i j} w_{d i},
$$

$a_{i j}$ - generalized coordinates which are to be determined,

$w_{i}(x)$ - functions making the basis for Galerkin procedure of the variational analysis,

$w_{q i}=w_{i}\left(x_{q}\right)$ - value of $w_{i}$ in place of the absorber attachment,

$w_{d i}$ - component of the absorber mass deviation.

The operator Eqs (4)-(7) is similar to the operator which is encountered in original system Eq. (1) or (3). Hence eigen-modes $\left(v_{j}(x), v_{d j}\right)$ fulfill a priori both main as well as natural boundary conditions. Therefore also functions $w_{i}(x)$ are recommended to be selected as eigen-modes of the operator similar to (3). In principle the choice could be wide. For instance the final eigenmodes of the operator Eqs (4)-(7) can be adopted whatever method of their determination has been used (slope deflection method, see e.g. [25], FEM, experiment, etc.). Another possibility represent eigen-modes of the operator Eqs (4)-(7) with constant coefficients in the length coordinate (keeping the boundary conditions). All intermediate selection is allowed as well. It can be shown, that any arbitrary choice of eigen-modes mentioned here represent a complete set in $n$-dimensional space of functions continuous almost everywhere along the length coordinate. 
Therefore the set of eigen-modes either $\left(v_{j, 0}(x), v_{d j, 0}\right)$ or $\left(w_{j}(x), w_{d j}\right)$ can be used as the Galerkin basis for a variational analysis of the system (1), see e.g. [26]. Application of such a basis enables to eliminate beforehand many problems of numerical instability namely regarding components with higher space derivatives in the area of the free edge where natural boundary condition are applied.

Let us substitute series Eqs (10) into the system Eq. (3) and evaluate the scalar product with the function $\left(v_{k}(x), v_{d k}\right)$. Some manipulations result in a new eigen value problem for values $\omega_{j, g}$ and vectors $\mathbf{a}_{j, g}=\left[a_{j 1, g}, . ., a_{j n, g}\right]^{T}, j=1, . ., n$ :

$$
\mathbf{K} \cdot \mathbf{a}_{j, g}+2 \mathrm{iB} \cdot \omega_{j, g} \mathbf{a}_{j, g}-\mathbf{M} \cdot \omega_{j, g}^{2} \mathbf{a}_{j, g}=0
$$

where the square symmetric matrices $\mathbf{K}, \mathbf{B}, \mathbf{M} \in \mathbb{R}^{n \times n}$ read:

$$
\begin{aligned}
& \mathbf{K}=\left[\int_{S} E J(x) w_{k}^{\prime \prime}(x) w_{j}^{\prime \prime}(x) \mathrm{d} x+C\left(w_{q k}-w_{d k}\right)\left(w_{q j}-w_{d j}\right)\right], \\
& \mathbf{B}=\left[\int_{S} b(x) w_{k}(x) w_{j}(x) \mathrm{d} x+b_{d}\left(w_{q k}-w_{d k}\right)\left(w_{q j}-w_{d j}\right)\right], \\
& \mathbf{M}=\left[\int_{S} \mu(x)(x) w_{k}(x) w_{j}(x) \mathrm{d} x+m\left(w_{q k}-w_{d k}\right)\left(w_{q j}-w_{d j}\right)\right] .
\end{aligned}
$$

The eigen vibration of a continuous system with a concentrated supplement is now defined in generalized coordinates $\mathbf{a}_{j, g}$ which can be regarded as columns of the square matrix: $\mathbf{a}_{g}=$ $\left[\mathbf{a}_{1, g}, \ldots, \mathbf{a}_{n, g}\right]$, where $\mathbf{a}_{g} \in \mathbb{R}^{n \times n}$. The mathematical model has a form which comply with that representing a system of $n$ concentrated masses. Individual degrees of freedom, however, are ordered in way corresponding with well separated increasing eigen-values. So the convergence can be controlled quite easily limiting appropriately the number of eigen-modes taken into account or simply selecting the upper limit of $n$ parameter.

In general matrices $\mathbf{K}, \mathbf{B}, \mathbf{M}$ are full. If the basis $w_{j}(x)$ is selected as to be eigen-modes of operator Eqs (4)-(7), i.e. $v_{j, 0}(x)=w_{j}(x)$, then matrices $\mathbf{K}, \mathbf{M}$ become diagonal with elements corresponding to Eqs (8), (9). At last if the damping is proportional also B degenerates to diagonal form and movement proceeds independently in individual eigen-forms.

\section{MODIFICATION OF MODAL PROPERTIES}

Matrices K, M in Eq. (11) can be diagonalized through pre-multiplication by the vector $\mathbf{a}_{j, g}^{T}$. The same operation doesn't diagonalize the matrix $\mathbf{B}$, unless the damping is proportional. When the B becomes diagonal, eigen-modes behave independently. Therefore Eq. (11) obviously implicates, that an interaction of eigen-modes $\mathbf{a}_{j, g}$ is given by non-proportional damping. Therefore when $\mathbf{K}, \mathbf{M}$ are already diagonalized, then a successive vanishing of the absolute value of the $\mathbf{B}$ matrix off-diagonal elements can be approximately regarded as an indicator of a justifiability of neglecting of the damping non-proportionality. Commonly known towers or chimneys even if designed using several materials (concrete, steel, composites) can be approximately considered as proportional. Provided the vibration absorber is installed, effect of non-proportionality should be taken into account. However the fact that the structure itself remains proportionally damped and the only component introducing the non-proportionality is the absorber can be reflected as a special type of perturbation. Moreover the tuning of the absorber 
is always related with one of eigen-values. So that the frequency portrait of the system differs predominantly from the basic configuration in the domain of the absorber eigen frequency. With increasing difference of structure eigen-values and that of the damper, the influence of effective non-proportionality decreases. This effect is markedly visible assessing changes of modal properties due to non-proportional component of the damping.

Let us cancel matrix B from Eq. (11) and evaluate eigen-values and eigen-modes of the non-damped system:

$$
\mathbf{K} \cdot \mathbf{a}_{j, 0}-\mathbf{M} \cdot \omega_{j, 0}^{2} \mathbf{a}_{j, 0}=0 \quad \text { or } \quad \mathbf{K} \cdot \mathbf{a}_{0}-\mathbf{M} \cdot \boldsymbol{\omega}_{0}^{2} \mathbf{a}_{0}=0
$$

and suppose that eigen-modes are normalized. Then pre-multiplication by the matrix $\mathbf{a}_{0}^{T}$ produces diagonal matrices:

$$
\mathbf{a}_{0}^{T} \mathbf{K} \mathbf{a}_{0}=\boldsymbol{\omega}_{0}^{2}=\operatorname{diag}\left[\omega_{1,0}^{2}, . ., \omega_{n, 0}^{2}\right], \quad \mathbf{a}_{0}^{T} \mathbf{M} \mathbf{a}_{0}=\mathbf{I} .
$$

Adopting the first order approximation of perturbation, we can write:

$$
\mathbf{B}=\mathbf{B}_{p}+\Delta \mathbf{B}
$$

where matrix $\mathbf{B}_{p}$ corresponds to proportional part of the damping. Employing the same multiplications as above, one obtains:

$$
\mathbf{a}_{0}^{T} \mathbf{B} \mathbf{a}_{0}=\mathbf{D}_{p}+\Delta \mathbf{D}, \quad \text { where: } \quad \mathbf{D}_{p}=\mathbf{a}_{0}^{T} \mathbf{B}_{p} \mathbf{a}_{0}, \quad \Delta \mathbf{D}=\mathbf{a}_{0}^{T} \Delta \mathbf{B} \mathbf{a}_{0} .
$$

Matrix $\mathbf{D}_{p}$ is diagonal with elements $D_{j j, p}$ and represents influence of the proportional part of the damping. Matrix $\Delta \mathrm{D}$ is symmetric with zero diagonal: $\Delta D_{j j}=0$.

The inverse assignment can be easily deduced. Indeed, one can write:

$$
\left(\mathbf{a}_{0}^{T}\right)^{-1} \mathbf{D}_{p} \mathbf{a}_{0}^{-1}=\mathbf{B}_{p}, \quad\left(\mathbf{a}_{0}^{T}\right)^{-1} \Delta \mathbf{D} \mathbf{a}_{0}^{-1}=\Delta \mathbf{B} .
$$

Regarding the second relation in Eqs (14) it is obvious: $\left(\mathbf{a}_{0}^{T}\right)^{-1}=\mathbf{M \mathbf { a } _ { 0 }}$ and $\mathbf{a}_{0}^{-1}=\mathbf{a}_{0}^{T} \mathbf{M} \mathbf{a}_{0}$ and so can be received:

$$
\mathbf{B}_{p}=\mathbf{M a}_{0} \mathbf{D}_{p} \mathbf{a}_{0}^{T} \mathbf{M}, \quad \Delta \mathbf{B}=\mathbf{M a}_{0} \Delta \mathbf{D a}_{0}^{T} \mathbf{M} .
$$

Reducing matrix $\mathbf{B}$ to $\mathbf{B}_{P}$, the equation Eq. (11) reads:

$$
\mathbf{K} \cdot \mathbf{a}_{j, p}+2 \mathbf{i B}_{p} \cdot \omega_{j, p} \mathbf{a}_{j, p}-\mathbf{M} \cdot \omega_{j, p}^{2} \mathbf{a}_{j, p}=0 .
$$

Equation can be diagonalized and therefore it represents $n$ independent scalar equations for unknown eigen-values $\omega_{j, p}$ of the proportionally damped system:

$$
\omega_{j, 0}^{2}+2 \mathrm{i} D_{j j, p} \omega_{j, p}-\omega_{j, p}^{2}=0,
$$

which yields immediately:

$$
\omega_{j, p}=\mathrm{i} D_{j j, p} \pm \sqrt{\omega_{j, 0}^{2}-D_{j j, p}^{2}},
$$

as it corresponds with classical literature. It should be reminded, that the damping in general is considered to be small, in order to fulfill the condition $\omega_{j, 0}^{2} \gg D_{j j, p}^{2}$. 
Eigen-vectors of undamped and proportionally damped systems are identical, so that it holds:

$$
\mathbf{a}_{p}=\mathbf{a}_{0}
$$

In accordance with the first order approximation of perturbation, see Eq. (15), analogously the eigen-values and eigen-modes of the real structure can be approximated:

$$
\omega_{j, g}=\omega_{j, p}+\Delta \omega_{j}, \quad \mathbf{a}_{j, g}=\mathbf{a}_{j, p}+\Delta \mathbf{a}_{j} .
$$

Revisiting Eq. (11) and taking into account Eqs (15) and (23), modified equation appears:

$$
\left[\mathbf{K}+2 \mathrm{i}\left(\mathbf{B}_{p}+\Delta \mathbf{B}\right)\left(\omega_{j, p}+\Delta \omega_{j}\right)-\mathbf{M}\left(\omega_{j, p}+\Delta \omega_{j}\right)^{2}\right] \cdot\left(\mathbf{a}_{j, 0}+\Delta \mathbf{a}_{j}\right)=0 .
$$

Let us pre-multiply Eq. (24) by the vector $\mathbf{a}_{j, 0}^{T}$. Making use the orthonormality of eigen-modes $\mathbf{a}_{j, 0}$ following Eqs (14), canceling identity Eq. (19) and keeping only terms of the first degree of perturbation, we obtain after some manipulation:

$$
\Delta \omega_{j}=-\frac{\omega_{j, p} \cdot \mathbf{a}_{j, 0}^{T} \cdot \Delta \mathbf{B} \cdot \mathbf{a}_{j, 0}}{\mathbf{a}_{j, 0}^{T}\left(\mathbf{B}_{p}+\mathrm{i} \omega_{j, p} \mathbf{M}\right) \mathbf{a}_{j, 0}} .
$$

Regarding the last relation Eq. (16), we learn that only diagonal elements of matrix $\Delta \mathbf{D}$ are referenced. As they are zeroes by definition, the increment $\Delta \omega_{j}$ vanishes. Hence it can be concluded that on the level of the first degree of perturbation the eigen-values of proportionally and non-proportionally damped system are identical. Therefore it holds:

$$
\Delta \omega_{j}=0 \quad \Longrightarrow \quad \omega_{j, g}=\omega_{j, p} .
$$

Let us pay attention now to interaction of eigen-modes of the non-proportionally damped system. Eigen-vectors of proportional (or non-damped) system make up a complete set in $\mathbb{R}^{n}$. Consequently a perturbation $\Delta \mathbf{a}_{j}$ of the eigen-mode can be approximated with any arbitrary accuracy by a linear combination of eigen-modes of the proportional or non-damped system:

$$
\Delta \mathbf{a}_{j}=\sum_{k=1}^{n} \alpha_{k j} \mathbf{a}_{k, 0}
$$

where $\alpha_{k j}$ are coefficients to be determined.

We recall Eq. (24). After canceling identity Eq. (19), omitting terms of higher order of perturbation than the first, one obtains at first:

$$
\left[\mathbf{K}+2 \mathrm{i} \mathbf{B}_{p} \omega_{j, p}-\mathbf{M} \omega_{j, p}^{2}\right] \cdot \Delta \mathbf{a}_{j}+\left[2 \mathrm{i}\left(\mathbf{B}_{p} \Delta \omega_{j}+\Delta \mathbf{B} \omega_{j, p}\right)-2 \omega_{j, p} \Delta \omega_{j} \mathbf{M}\right] \cdot \mathbf{a}_{j, 0}=0 .
$$

Subsequently the series Eq. (27) is to be substituted into Eq. (28) and then the result premultiplied by $\mathbf{a}_{i, 0}^{T}$. Also consider Eq. (26). We read now:

$$
\mathbf{a}_{i, 0}^{T} \cdot\left(\mathbf{K}+2 \mathbf{i} \mathbf{B}_{p} \omega_{j, p}-\mathbf{M} \omega_{j, p}^{2}\right) \cdot \sum_{k=1}^{n} \alpha_{k j} \mathbf{a}_{k, 0}+2 \mathrm{i} \omega_{j, p} \mathbf{a}_{i, 0}^{T} \cdot \Delta \mathbf{B} \cdot \mathbf{a}_{j, 0}=0
$$

Some manipulation respecting to Eq. (14) provides coefficients $\alpha_{k j}$ :

$$
\alpha_{k j}=-\frac{2 \mathrm{i} \omega_{j, p} \Delta B_{k j}}{\omega_{j, 0}^{2}+2 \mathrm{i} \omega_{j, p} B_{k k, p}-\omega_{j, p}^{2}}, \quad \alpha_{k k}=0 .
$$


Assembling Eqs (23), (27) and (30) together, final expression for perturbed eigen-modes can be written:

$$
\mathbf{a}_{j, g}=\mathbf{a}_{j, 0}-\sum_{k=1}^{n} \frac{2 \mathrm{i} \omega_{j, p} \Delta B_{k j} \cdot \mathbf{a}_{k, 0}}{\omega_{j, 0}^{2}+2 \mathrm{i} \omega_{j, p} B_{k k, p}-\omega_{j, p}^{2}} .
$$

Take a note that the $j$-th member of summation above vanishes and doesn't influence the basic term $\mathbf{a}_{j, p}$. Coefficients Eq. (30) demonstrate the rate of other eigen-modes modifying the shape of the $j$-th eigen-mode. It is obvious that this phenomenon decays with vanishing $\Delta \mathbf{B}$ matrix.

Let us get back to series Eqs (10). They have been introduced dealing with non-damped system. However actual vectors $\mathbf{a}_{j, g}$ corresponds with the initial system Eq. 3 . Consequently the eigen-modes of the initial problem can be formulated as follows:

$$
v_{j, g}(x)=\sum_{i=1}^{n} a_{i j, g} w_{i}(x), \quad v_{q, g}=\sum_{i=1}^{n} a_{i j, g} w_{q i}, \quad v_{d, g}=\sum_{i=1}^{n} a_{i j, g} w_{d i} .
$$

Regarding the time coordinate of individual eigen-modes, it is given by exponential $\exp (\mathrm{i} \omega t)$ introduced in Eqs (2), where $\omega$ should be replaced by $\omega_{j, p}$, see Eq. (21).

\section{NUMERICAL EXPERIMENTS}

The accuracy of approximate solutions of modal properties has been investigated for a linear discrete numerical model of the existing TV tower equipped with a tuned mass damper. The tower height is $185 \mathrm{~m}$. It consists of the lower part made of reinforced concrete (square crosssection with a side length $7,8 \mathrm{~m}$, thickness $0,6 \mathrm{~m}$ and length $51,1 \mathrm{~m}$ ) followed by a variable cross-section steel cylinders with diameters of $6 \mathrm{~m}$ (thickness $25 \mathrm{~mm}$, length 19,5 m), 4,5 m (thickness $25 \mathrm{~mm}$, length 41,8 m), $3 \mathrm{~m}$ (thickness $22 \mathrm{~mm}$, length $24 \mathrm{~m}$ ) and 1,9 $\mathrm{m}$ (thickness $20 \mathrm{~mm}$, length $28 \mathrm{~m}$ ). The top of the tower creates a laminate extension with a diameter of $1,9 \mathrm{~m}$ (thickness $14 \mathrm{~mm}$, length 22,6 m). The tower is in the heel fully clamped into subsoil. The pendulum absorber supplemented with viscous dampers has been installed into a laminate extension at a top of the tower to suppress excessive vibrations induced by a vortex-shedding and other aero-elastic effects. The absorber significantly eliminates unacceptable stresses in the structure, which are dangerous from the point of view of a material fatigue and a life-time of the structure. The absorber has been designed and tuned to be the most effective at vibrations related with the second eigen-mode, i.e. second eigen-value of the tower. The mass of the absorber is 1 tone, which is roughly $1 / 10$ of a generalized mass of the second eigen-mode. A part of a vibration energy of the structure is transformed into heat due to viscous dampers of the absorber. In order to reach the most effective behavior of the absorber, its damping is intentionally higher than damping properties of the structure itself. In our case the damping ratio of the structure has been experimentally determined by low value equal $0,5 \%$, while the ideal damping ratio of absorber has been calculated and set equal to $20 \%$. This difference could generate the significant source of non-proportionality of the damping.

The analyzed two dimensional discrete numerical model of the tower has been created in CALFEM (MATLAB toolbox intended for FEM analysis). The model without tuned mass damper has been built using beam elements and consisted of 15 nodes. Each node had 2 degrees of freedom, e.g. horizontal displacement and rotation around horizontal axis. The absorber has been subsequently modeled as a concentrated mass connected to the top of the tower with Kelvin-Voigt damping term having one degree of freedom in horizontal direction. Supplementing one degree of freedom due to absorber, a total number of eigen-modes of the whole system raised by one. 
The new eigen-mode is associated with the second eigen-mode of the tower with respect to tuning of the absorber. The completed structure has now two eigen-modes which are similar in shape but completely different in phase shift between the tower top and absorber, see Figure 1.

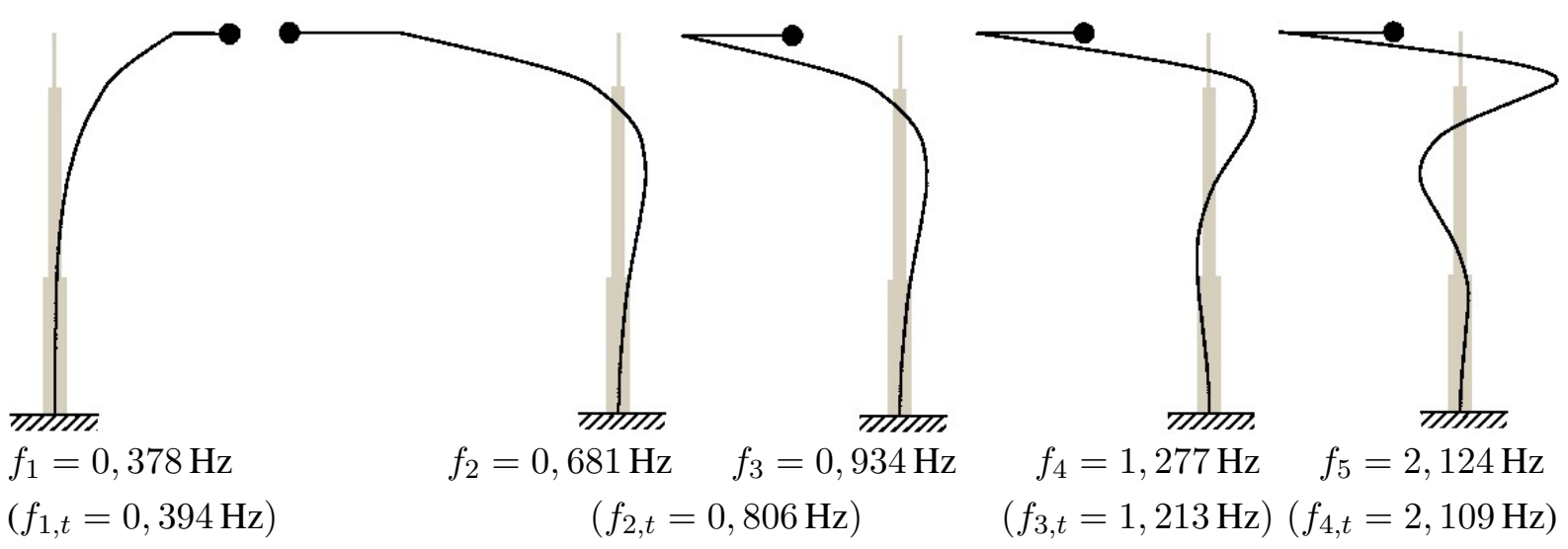

Figure 1: The first five eigen-modes of the tower with absorber; eigen-frequencies of the tower with and without absorber $\left(f_{i}\right.$ and $\left.f_{i, t}\right)$ are below corresponding eigen-modes.

The numerical model has been analyzed in order to determine a suitability of proposed perturbation method for the calculation of eigen-values and eigen-modes of the non-proportionally damped slender structure with the absorber. The damping matrix of the tower itself has been set to be proportional to a combination of the mass and the stiffness matrix. Multiplicative coefficients related to these stiffness and mass matrices have been calculated from the given structural damping ratio $(\zeta=0,005)$ for the first two eigen-values. Factor of the damping nonproportionality of the system has been examined as follows: a set of various damping ratios of the absorber has been used $\left(\zeta_{d}=0 \div 0,8\right)$, while constant damping matrix of the tower has been kept. The first six eigen-modes of undamped tower with absorber have been used as a Galerkin base $\left(w_{j}(x), w_{d j}\right)$ for a reduction of the matrix system Eq. (1) using transformation (10). After the reduction the modal properties have been evaluated according Eqs (26 or 21) and (32). The results have been subsequently compared with the exact solution obtained using the complex subspace iteration method, see [19].

In Figure 2 the exact eigen-values and approximate eigen-values of the tower with the absorber as a function of $\zeta_{d}$ are depicted in the complex plane. The solid curves represent the exact solution, while the dashed curves the approximate one. The identical markers on both curves correspond to the identical damping ratios of the absorber. Changes of eigen-value positions indicate obviously, that the damping of the absorber strongly influences the second, third and fourth eigen-values. On the other hand the first and sixth eigen-values are affected imperceptibly. The influence of the absorber on higher eigen-values significantly decreases with the increasing distance between eigen-value of the absorber and respective eigen-value of the structure. To quantify an accuracy of the perturbation method the relative error in the determination of eigen-values $\omega_{j, g}$ has been calculated separately for imaginary and real parts as follows:

$$
\begin{aligned}
& \delta_{\operatorname{Re}}\left(\omega_{j, g}\right)=\frac{\operatorname{Re}\left(\omega_{j, p}\right)-\operatorname{Re}\left(\omega_{j, g(e x)}\right)}{\operatorname{Re}\left(\omega_{j, g(e x)}\right)} \cdot 100[\%], \\
& \delta_{\operatorname{Im}}\left(\omega_{j, g}\right)=\frac{\operatorname{Im}\left(\omega_{j, p}\right)-\operatorname{Im}\left(\omega_{j, g(e x)}\right)}{\operatorname{Im}\left(\omega_{j, g(e x)}\right)} \cdot 100[\%] .
\end{aligned}
$$

where $\omega_{j, g(e x)}$ is an exact eigen-value of the non-proportionally damped tower with absorber. 

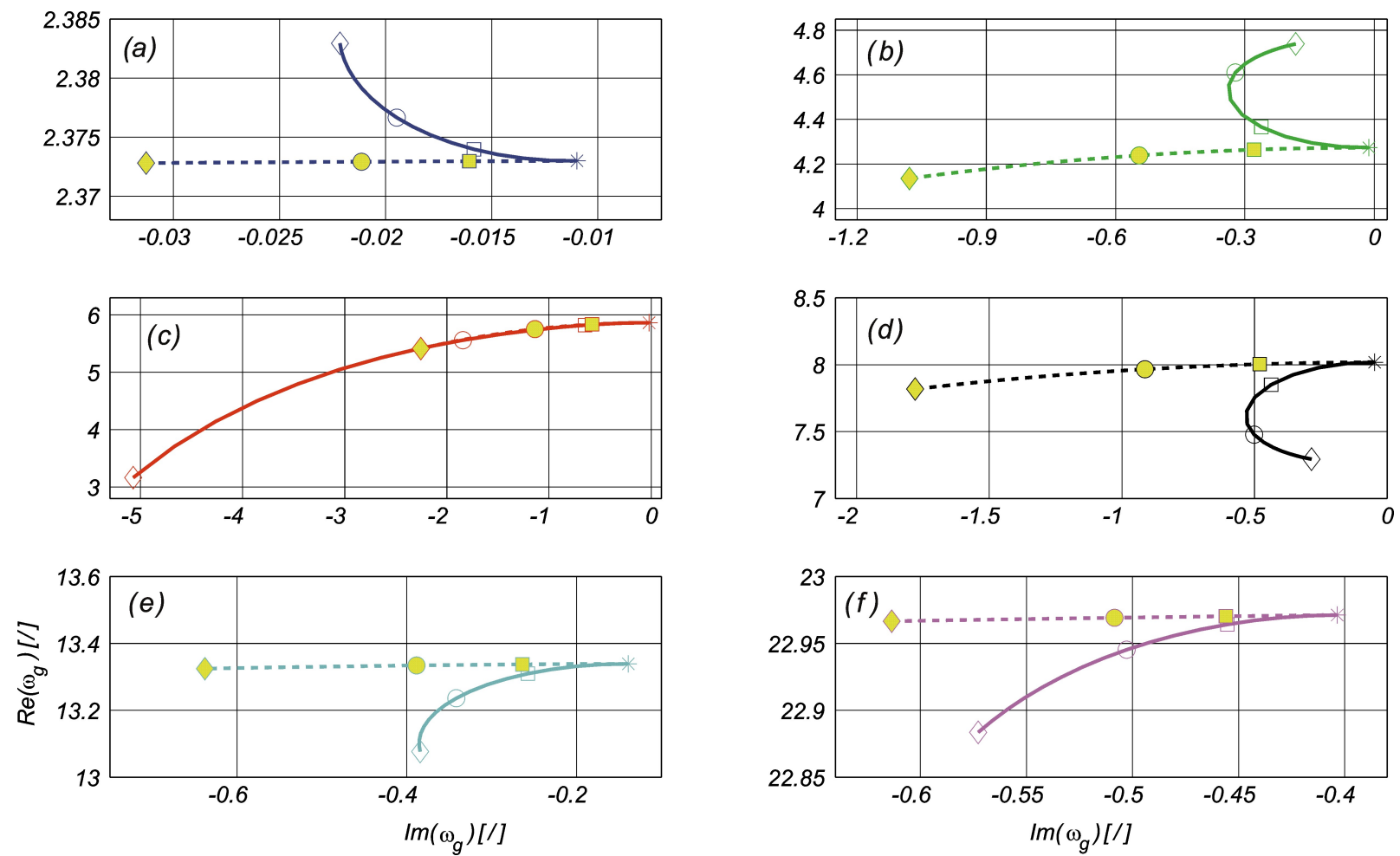

Figure 2: The first six eigen-values $\omega_{j, g}$ as a function of damping ratio $\zeta_{d}$ (solid line - exact solution; dashed line and marker with yellow fill - approximate solution)

$$
\left(*-\zeta_{d}=0 ; \square-\zeta_{d}=0,2 ; \circ-\zeta_{d}=0,4 ; \diamond-\zeta_{d}=0,8\right) \text {. }
$$

The calculated relative errors for the selected damping ratio $\zeta_{d}$ are summarized in Tables 1 and 2. The errors in both the imaginary and the real parts of eigen-values are increasing with increase in $\zeta_{d}$. They emerge due to rising difference between structural damping ratios and damping ratio $\zeta_{d}$. The error of the real part representing the "frequency" component of an eigen-value, is substantially lower than that of the imaginary part representing the eigen-value "damping" component. The substantial errors came up for eigen-values which are close to eigen-value of the absorber, in particular for the second to fourth eigen-value. For the realistic damping ratio $\zeta_{d}$ being $20 \%$ the relative errors in determination of the real parts are at most $2 \%$ and $11 \%$ concerning the imaginary part. This accuracy is acceptable and consequently the perturbation strategy outlined in the second and third chapters is entitled to assess dynamic behavior of existing structures provided with one passive damping element. In our case a limiting value of $\zeta_{d}$ for which the errors are reasonable is equal 30\%. Beyond this value the errors notably of the imaginary part could be higher than $25 \%$ and more sophisticated approach should be employed.

The mechanical interaction between real eigen-modes of the tower is reflected by non-zero off-diagonal elements of the matrix, where columns comprise approximate complex eigenmodes $\mathbf{a}_{j, g}$ of reduced model, Eq. 11. The complex element $a_{i j, g}$ represents the contribution of the $\mathrm{i}$-th real eigen-mode to the complex and real part of the $\mathrm{j}$-th eigen-mode of the non-proportionally damped system. Similarly to the analysis of complex eigen-values also the vectors $\mathbf{a}_{j, g}$ have been evaluated for several damping ratios $\zeta_{d}$, see Tables 3, 4, 5 and 6 . For zero $\zeta_{d}$ the complex eigen-modes of the whole system $v_{j, g}(x)$ are almost identical with the real 


\begin{tabular}{ccccccc}
\hline$\zeta_{d}$ & \multicolumn{6}{c}{$\omega_{j, g}$} \\
\cline { 2 - 7 }$[/]$ & $\mathrm{j}=1$ & 2 & 3 & 4 & 5 & 6 \\
\cline { 2 - 7 } 0 & $-0,0001$ & $-0,0015$ & 0,0006 & 0,0010 & 0,0001 & $1,04 \mathrm{e}-5$ \\
0,1 & $-0,0099$ & $-0,5409$ & 0,0219 & 0,4617 & 0,0514 & 0,0064 \\
0,2 & $-0,0427$ & $-2,3201$ & 0,2202 & 1,9590 & 0,2071 & 0,0265 \\
0,3 & $-0,0939$ & $-5,2247$ & 1,0639 & 4,3440 & 0,4468 & 0,0595 \\
0,4 & $-0,1574$ & $-8,0590$ & 3,4599 & 6,5392 & 0,7406 & 0,1041 \\
0,8 & $-0,4255$ & $-12,7311$ & 71,1439 & 7,1908 & 1,8949 & 0,3632
\end{tabular}

Table 1: Percentage relative error $\delta_{\operatorname{Re}}\left(\omega_{j, g}\right)$.

\begin{tabular}{ccccccc}
\hline$\zeta_{d}$ & \multicolumn{6}{c}{$\omega_{j, g}$} \\
\cline { 2 - 7 }$[/]$ & $\mathrm{j}=1$ & 2 & 3 & 4 & 5 & 6 \\
\cline { 2 - 7 } 0 & $-0,0004$ & $-0,0015$ & 0,0035 & $-0,0009$ & $-3.7 \mathrm{e}-5$ & $-1.3 \mathrm{e}-6$ \\
0,1 & 0,1770 & 1,1026 & $-2,3494$ & 1,7488 & 0,3832 & 0,0199 \\
0,2 & 1,3776 & 6,4283 & $-10,6109$ & 9,7805 & 2,4708 & 0,1583 \\
0,3 & 4,1112 & 23,5600 & $-24,3681$ & 31,6932 & 6,8460 & 0,5099 \\
0,4 & 8,5116 & 69,2541 & $-38,2283$ & 82,1212 & 13,7089 & 1,1419 \\
0,8 & 41,2246 & 491,9443 & $-55,5150$ & 524,4971 & 66,0251 & 7,1132
\end{tabular}

Table 2: Percentage relative error $\delta_{\operatorname{Im}}\left(\omega_{j, g}\right)$.

eigen-modes $v_{j, 0}(x)$ and the contributions of other eigen-modes i.e. elements $a_{i j, g}$ are nearly vanishing, see Table 3. For higher values of $\zeta_{d}$ the interaction in particular of the second, third and fourth eigen-modes are important and not negligible, see Tables 4, 5 and 6, where the significant values are typed in bold. It comes from the fact, that these eigen-modes are very close in their eigen-frequencies and also regarding the tuning frequency of the absorber. The shapes of complex eigen-modes for realistic $\zeta_{d}=0,2$ and $\zeta_{d}=0,8$ are depicted in Figures 3 and 4 respectively. Only the perturbed solution is presented in Figure 3 due to weak graphic resolution of exact and approximate solutions, which implies a good accuracy of the latter one. However the accuracy is dropping with increasing damping ratio. Finally the approximate process fails when the damping ratio falls into the interval $\zeta_{d} \in(0.4-0.8)$, as it is notably visible referring particularly the fourth eigen-mode, see Figure 4.

In case of realistic $\zeta_{d}$ the considerable out of phase character of the second to fourth complex eigen-modes reflects the mechanical interaction of their corresponding undamped real counterparts. Consequently the zero nodes moving along the vertical axis of the structure emerge and vanish during their eigen-vibration process. On the other hand the first, fifth and sixth complex eigen-modes are very close to their undamped real eigen-modes and thus each one is almost synchronous. The mechanical interaction is in our case more evident and strong with increasing of the damping ratio $\zeta_{d}$ above the structural damping ratios. However for high value $\zeta_{d}=0,8$ it appears that although the interaction is significant, some eigen-modes such as the second and the third are much closer to the corresponding real modes than in case of lower $\zeta_{d}$. So we can ascertain that there exists a ratio $\zeta_{d, e x t r}$ leading to the highest phase shift variability within the eigen-modes, which is dropping with $\zeta_{d}$ approaching 0 or 1. 

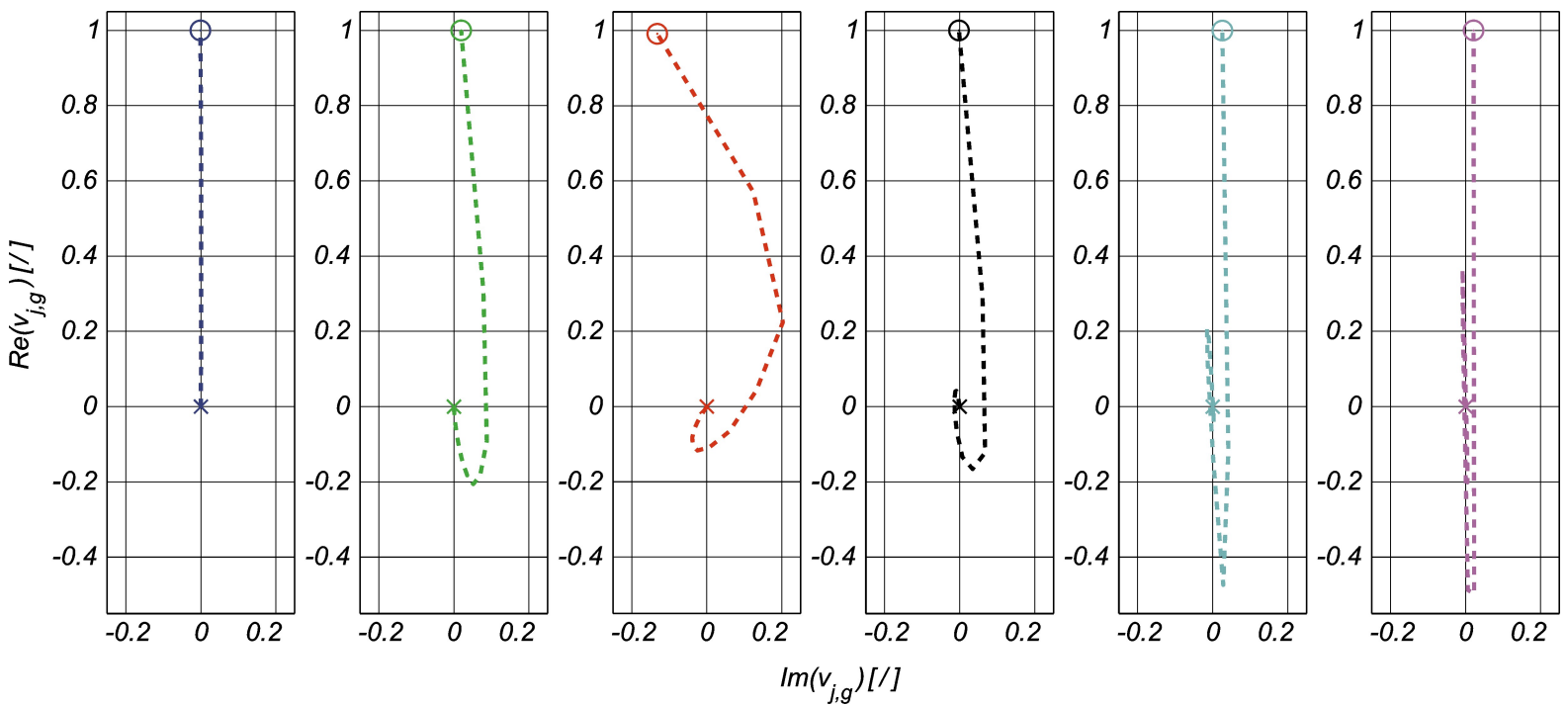

Figure 3: The first six eigen-mode of the shaft of the tower in the complex plane $\left(\zeta_{d}=0,2\right)$ (Approximate solution; $\mathrm{x}$ - heel of the tower, $\circ$ - approximate coordinate of the tower top).
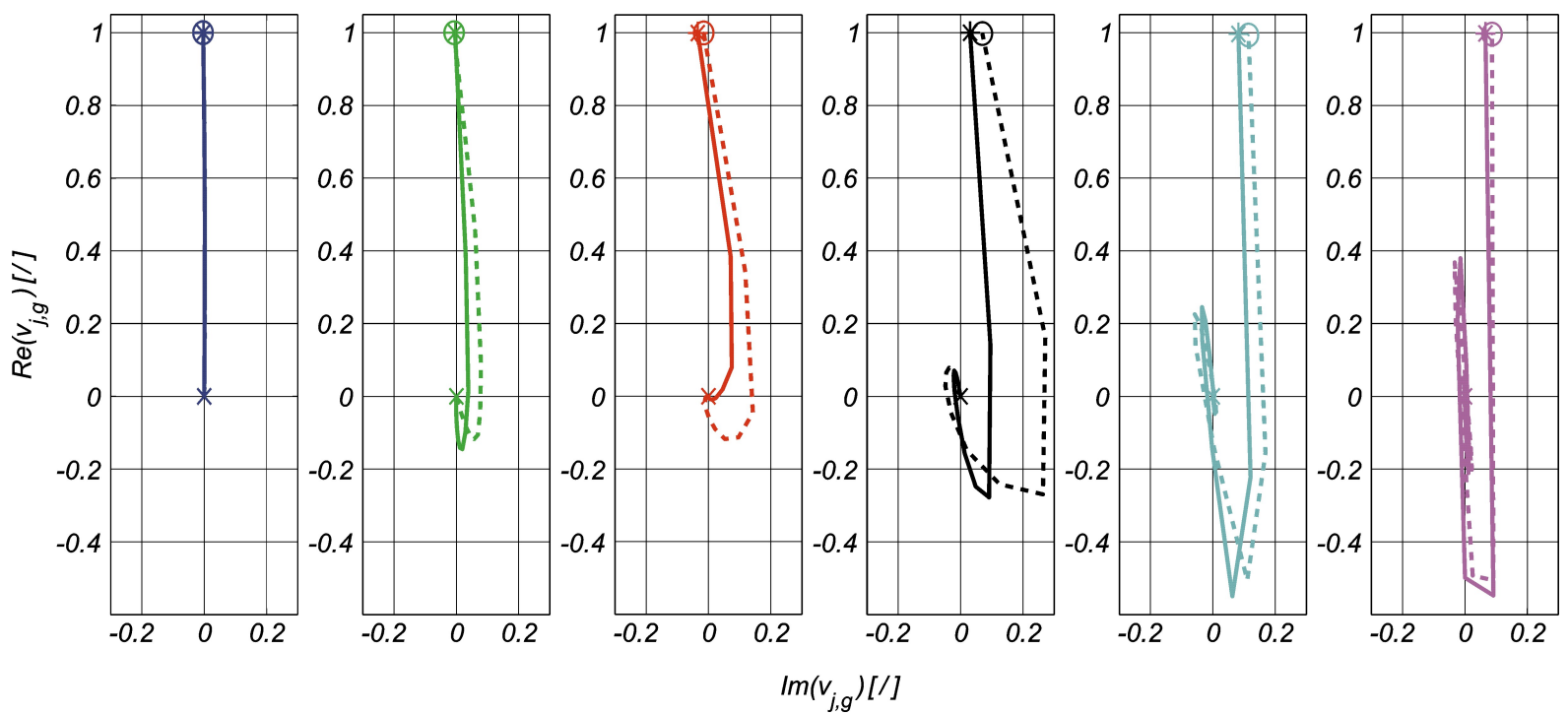

Figure 4: The first six eigen-mode of the shaft of the tower in the complex plane $\left(\zeta_{d}=0,8\right)$

(solid line - exact solution; dashed line - approximate solution;

$\mathrm{x}$ - heel of the tower, $\circ$ and $\star$ - approximate and exact coordinate of the tower top).

The important aspect from the point of view of complex eigen-modes is the moment when the real part of the third eigen-value vanishes and respective transformation of the corresponding eigen-mode from the complex to the real domain takes place. 


\begin{tabular}{|c|c|c|c|c|c|c|}
\hline \multirow[t]{2}{*}{$i$} & \multicolumn{6}{|c|}{$a_{i j, g}$} \\
\hline & $\mathrm{j}=1$ & 2 & 3 & 4 & 5 & 6 \\
\hline 1 & 1 & $-3 e-6+2 e-3 i$ & $4 e-7+1 e-3 i$ & $2 e-6+4 e-4 i$ & $9 e-7+9 e-5 i$ & $5 e-7+3 e-5 i$ \\
\hline 2 & $4 e-6-9 e-4 i$ & 1 & $-2 e-5-8 e-3 i$ & $-2 e-5-3 e-3 i$ & $-7 e-6-6 e-4 i$ & $-4 e-6-2 e-4 i$ \\
\hline 3 & $1 e-6-4 e-4 i$ & $8 e-6+6 e-3 i$ & 1 & $-5 e-5-6 e-3 i$ & $-1 e-5-10 e-3 i$ & $-6 e-6-3 e-4 i$ \\
\hline 4 & $2 e-7-1 e-4 i$ & $5 e-6+2 e-3 i$ & $3 e-5+4 e-3 i$ & 1 & $-1 e-5-1 e-3 i$ & $-5 e-6-3 e-4 i$ \\
\hline 5 & $2 e-8-2 e-5 i$ & $8 e-7+2 . e-4 i$ & $3 e-6+4 e-4 i$ & $5 e-6+6 e-4 i$ & 1 & $-4 e-6-2 \cdot e-4 i$ \\
\hline 6 & $3 e-9-3 e-6 i$ & $1 e-7+4 e-5 i$ & $4 e-7+8 e-5 i$ & $6 e-7+1 e-4 i$ & $1 e-6+1 e-4 i$ & 1 \\
\hline
\end{tabular}

Table 3: Contribution $a_{i j, g}$ of real eigen-modes to individual complex eigen-modes for $\zeta_{d}=0$.

\begin{tabular}{ccccccc}
\hline$i$ & \multicolumn{5}{c}{$a_{i j, g}$} \\
\cline { 2 - 7 } & $\mathrm{j}=1$ & 2 & 3 & 4 & 5 & 6 \\
\cline { 2 - 7 } & 1 & $-3-\mathrm{e} 3-0,02 \mathrm{i}$ & $-3 \mathrm{e}-3-0,02 \mathrm{i}$ & $-8 \mathrm{e}-4-0,01 \mathrm{i}$ & $-7 \mathrm{e}-5-4 \mathrm{e}-3 \mathrm{i}$ & $-3 \mathrm{e}-5-1 \mathrm{e}-3 \mathrm{i}$ \\
2 & $+1 \mathrm{e}-3+0,01 \mathrm{i}$ & 1 & $\mathbf{0 , 0 3}+\mathbf{0 , 2 7 i}$ & $\mathbf{1 e - 3 + 0 , 1 2 i}$ & $-7 \mathrm{e}-4+0,03 \mathrm{i}$ & $-4 \mathrm{e}-5+0,01 \mathrm{i}$ \\
3 & $+7 \mathrm{e}-4+8 \mathrm{e}-3 \mathrm{i}$ & $\mathbf{- 0 , 0 2}-\mathbf{- 0 , 2 0 i}$ & 1 & $\mathbf{- 0 , 0 3 + 0 , 2 6 i}$ & $-4 \mathrm{e}-3+0,05 \mathrm{i}$ & $-5 \mathrm{e}-4+0,02 \mathrm{i}$ \\
4 & $1 \mathrm{e}-4+4 \mathrm{e}-3 \mathrm{i}$ & $2 \mathrm{e}-3-0,06 \mathrm{i}$ & $\mathbf{0 , 0 3}-\mathbf{0 , 1 9 i}$ & 1 & $-4 \mathrm{e}-3+0,05 \mathrm{i}$ & $-3 \mathrm{e}-4+0,01 \mathrm{i}$ \\
5 & $4 \mathrm{e}-8+7 \mathrm{e}-4 \mathrm{i}$ & $6 \mathrm{e}-4-0,01 \mathrm{i}$ & $3 \mathrm{e}-3-0,02 \mathrm{i}$ & $3 \mathrm{e}-3-0,03 \mathrm{i}$ & 1 & $6 \mathrm{e}-5+0,01 \mathrm{i}$ \\
6 & $-4 \mathrm{e}-7+1 \mathrm{e}-4 \mathrm{i}$ & $1 \mathrm{e}-4-2 \mathrm{e}-3 \mathrm{i}$ & $4 \mathrm{e}-4-4 \mathrm{e}-3 \mathrm{i}$ & $3 \mathrm{e}-4-5 \mathrm{e}-3 \mathrm{i}$ & $3 \mathrm{e}-5-6 \mathrm{e}-3 \mathrm{i}$ & 1
\end{tabular}

Table 4: Contribution $a_{i j, g}$ of real eigen-modes to individual complex eigen-modes for $\zeta_{d}=0,2$.

\begin{tabular}{ccccccc}
\hline$i$ & \multicolumn{5}{c}{$a_{i j, g}$} \\
\cline { 2 - 7 } & $\mathrm{j}=1$ & 2 & 3 & 4 & 5 & 6 \\
\cline { 2 - 7 } & 1 & $-0,01-0,05 \mathrm{i}$ & $-0,01-0,04 \mathrm{i}$ & $-3 \mathrm{e}-3-0,03 \mathrm{i}$ & $-2 \mathrm{e}-4-8 \mathrm{e}-3 \mathrm{i}$ & $-6 \mathrm{e}-5-3 \mathrm{e}-3 \mathrm{i}$ \\
2 & $5 \mathrm{e}-3+0,03 \mathrm{i}$ & 1 & $\mathbf{0 , 1 3}+\mathbf{0 , 5 3 i}$ & $\mathbf{3 e - 3 + 0 , 2 4 i}$ & $-3 \mathrm{e}-3+0,06 \mathrm{i}$ & $-5 \mathrm{e}-4+0,02 \mathrm{i}$ \\
3 & $3 \mathrm{e}-3+0,02 \mathrm{i}$ & $\mathbf{- 0 , 0 7}-\mathbf{- 0 , 3 9 i}$ & 1 & $\mathbf{- 0 , 1 2 + 0 , 5 0 i}$ & $-0,02+0,09 \mathrm{i}$ & $-3 \mathrm{e}-3+0,03 \mathrm{i}$ \\
4 & $5 \mathrm{e}-4+7 \mathrm{e}-3 \mathrm{i}$ & $7 \mathrm{e}-3-0,13 \mathrm{i}$ & $\mathbf{0 , 1 0}-\mathbf{0 , 3 6 i}$ & 1 & $-0,02+0,11 \mathrm{i}$ & $-2 \mathrm{e}-3+0,03 \mathrm{i}$ \\
5 & $2 \mathrm{e}-6+1 \mathrm{e}-3 \mathrm{i}$ & $3 \mathrm{e}-3-0,02 \mathrm{i}$ & $0,01-0,04 \mathrm{i}$ & $0,01-0,06 \mathrm{i}$ & 1 & $-1 \mathrm{e}-4+0,02 \mathrm{i}$ \\
6 & $-1 \mathrm{e}-6+3 \mathrm{e}-4 \mathrm{i}$ & $5 \mathrm{e}-4-4 \mathrm{e}-3 \mathrm{i}$ & $2 \mathrm{e}-3-8 \mathrm{e}-3 \mathrm{i}$ & $1 \mathrm{e}-3-0,01 \mathrm{i}$ & $2 \mathrm{e}-4-0,01 \mathrm{i}$ & 1
\end{tabular}

Table 5: Contribution $a_{i j, g}$ of real eigen-modes to individual complex eigen-modes for $\zeta_{d}=0,4$.

\begin{tabular}{ccccccc}
\hline$i$ & \multicolumn{5}{c}{$a_{i j, g}$} \\
\cline { 2 - 7 } & $\mathrm{j}=1$ & 2 & 3 & 4 & 5 & 6 \\
\cline { 2 - 7 } & 1 & $0,04-0,08 \mathrm{i}$ & $-0,04-0,07 \mathrm{i}$ & $-0,01-0,05 \mathrm{i}$ & $-7 \mathrm{e}-4-0,02 \mathrm{i}$ & $-1 \mathrm{e}-4-6 \mathrm{e}-3 \mathrm{i}$ \\
2 & $0,02+0,05 \mathrm{i}$ & 1 & $\mathbf{0 , 4 9 + 0 , 9 6 i}$ & $\mathbf{0 , 0 1 + 0 , 4 8 i}$ & $-0,01+0,12 \mathrm{i}$ & $-3 \mathrm{e}-3+0,04 \mathrm{i}$ \\
3 & $0,01+0,03 \mathrm{i}$ & $\mathbf{- 0 , 2 8}-\mathbf{0 , 7 3 i}$ & 1 & $\mathbf{- 0 , 4 3 + 0 , 8 7 i}$ & $-0,06+0,17 \mathrm{i}$ & $-0,01+0,06 \mathrm{i}$ \\
4 & $2 \mathrm{e}-3+0,01 \mathrm{i}$ & $0,03-0,25 \mathrm{i}$ & $\mathbf{0 , 3 7 - 0 , 6 0 i}$ & 1 & $-0,06+0,20 \mathrm{i}$ & $-8 \mathrm{e}-3+0,06 \mathrm{i}$ \\
5 & $1 \mathrm{e}-5+3 \mathrm{e}-3 \mathrm{i}$ & $0,01-0,04 \mathrm{i}$ & $0,04-0,07 \mathrm{i}$ & $0,05-0,12 \mathrm{i}$ & 1 & $-1 \mathrm{e}-3+0,04 \mathrm{i}$ \\
6 & $-5 \mathrm{e}-6+6 \mathrm{e}-4 \mathrm{i}$ & $2 \mathrm{e}-3-8 \mathrm{e}-3 \mathrm{i}$ & $7 \mathrm{e}-3-0,01 \mathrm{i}$ & $5 \mathrm{e}-3-0,02 \mathrm{i}$ & $1 \mathrm{e}-3-0,02 \mathrm{i}$ & 1
\end{tabular}

Table 6: Contribution $a_{i j, g}$ of real eigen-modes to individual complex eigen-modes for $\zeta_{d}=0,8$. 
The complexity or phase shift variability in length coordinate of the eigen-modes could be assessed using a modal phase co-linearity criterion defined in [27] as follows:

$$
\operatorname{MPC}\left(v_{j, g}(x)\right)=\left(\frac{\lambda_{1}-\lambda_{2}}{\lambda_{1}+\lambda_{2}}\right)^{2} .
$$

where

$$
\begin{gathered}
\lambda_{1,2}=\frac{S_{x x}+S_{y y}}{2} \pm S_{x y} \sqrt{\eta^{2}+1} . \\
\eta=\frac{S_{y y}-S_{x x}}{2 S_{x y}} . \\
S_{x x}=\operatorname{Re}\left(v_{j, g}(x)\right)^{T} \cdot \operatorname{Re}\left(v_{j, g}(x)\right), \\
S_{y y}=\operatorname{Im}\left(v_{j, g}(x)\right)^{T} \cdot \operatorname{Im}\left(v_{j, g}(x)\right), \\
S_{x y}=\operatorname{Re}\left(v_{j, g}(x)\right)^{T} \cdot \operatorname{Im}\left(v_{j, g}(x)\right) .
\end{gathered}
$$

It uses the variance and covariance of the real and imaginary part of the vector Eq. (37). Parameter MPC ranges from 0 to 1 . The unity holds for fully phased vector (no phase shift), whereas the zero for totally dispersed vector in the complex plane. The MPC parameter has been evaluated for each complex eigen-mode and ratio $\zeta_{d}$ and written out in Tables 7 or 8 regarding exact or approximate solutions respectively.

\begin{tabular}{ccccccc}
\hline$\zeta_{d}$ & \multicolumn{6}{c}{$1-\mathrm{MPC}_{e x}\left(v_{j, g}(x)\right)$} \\
\cline { 2 - 7 }$[/]$ & $\mathrm{j}=1$ & 2 & 3 & 4 & 5 & 6 \\
\cline { 2 - 7 } 0 & $3 \mathrm{e}-7$ & 0,0001 & 0,0003 & $2 \mathrm{e}-5$ & $4 \mathrm{e}-6$ & $9 \mathrm{e}-7$ \\
0,1 & $1 \mathrm{e}-6$ & 0,0207 & 0,1014 & 0,0087 & 0,0026 & 0,0006 \\
0,2 & $7 \mathrm{e}-6$ & 0,0589 & 0,2493 & 0,0359 & 0,0104 & 0,0024 \\
0,3 & $2 \mathrm{e}-5$ & 0,0682 & 0,2754 & 0,0771 & 0,0221 & 0,0054 \\
0,4 & $2 \mathrm{e}-5$ & 0,0496 & 0,2364 & 0,1102 & 0,0355 & 0,0093 \\
0,8 & $5 \mathrm{e}-5$ & 0,0121 & 0,0493 & 0,0734 & 0,0728 & 0,0294
\end{tabular}

Table 7: Reverse value of modal phase co-linearity criterion for exact complex eigen-modes.

\begin{tabular}{ccccccc}
\hline$\zeta_{d}$ & \multicolumn{6}{c}{$1-\mathrm{MPC}_{a p p}\left(v_{j, g}(x)\right)$} \\
\cline { 2 - 7 }$[/]$ & $\mathrm{j}=1$ & 2 & 3 & 4 & 5 & 6 \\
\cline { 2 - 7 } 0 & $3 \mathrm{e}-7$ & 0,0001 & 0,0003 & $2 \mathrm{e}-5$ & $4 \mathrm{e}-6$ & $1 \mathrm{e}-6$ \\
0,1 & $1 \mathrm{e}-6$ & 0,0218 & 0,0984 & 0,0084 & 0,0027 & 0,0007 \\
0,2 & $9 \mathrm{e}-6$ & 0,0737 & 0,2483 & 0,0348 & 0,0111 & 0,0027 \\
0,3 & $2 \mathrm{e}-5$ & 0,1188 & 0,2989 & 0,0784 & 0,0250 & 0,0062 \\
0,4 & $3 \mathrm{e}-5$ & 0,1390 & 0,2805 & 0,1372 & 0,0442 & 0,0111 \\
0,8 & 0,0001 & 0,0840 & 0,1514 & 0,4396 & 0,1624 & 0,0434
\end{tabular}

Table 8: Reverse value of modal phase co-linearity criterion for approximate complex eigen-modes. 
To compare the approximate and the exact solution of complex eigen-modes the relative error using this criterion has been evaluated. The error has been assumed in a form:

$$
\Delta \operatorname{MPC}\left(v_{j, g}(x)\right)=\frac{\operatorname{MPC}_{a p p}\left(v_{j, g}(x)\right)-\operatorname{MPC}_{e x}\left(v_{j, g}(x)\right)}{\operatorname{MPC}_{e x}\left(v_{j, g}(x)\right)} \cdot 100[\%] .
$$

\begin{tabular}{ccccccc}
\hline$\zeta_{d}$ & \multicolumn{6}{c}{$\Delta \mathrm{MPC}\left(v_{j, g}(x)\right)$} \\
\cline { 2 - 7 }$[/]$ & $\mathrm{j}=1$ & 2 & 3 & 4 & 5 & 6 \\
\cline { 2 - 7 } 0 & $-6 \mathrm{e}-7$ & $-2 \mathrm{e}-5$ & 0,0006 & $5 \mathrm{e}-5$ & $-5 \mathrm{e}-6$ & $-1 \mathrm{e}-5$ \\
0,1 & $-3 \mathrm{e}-5$ & $-0,1163$ & 0,3322 & 0,0298 & $-0,0061$ & $-0,0067$ \\
0,2 & $-0,0002$ & $-1,5777$ & 0,1338 & 0,1064 & $-0,0662$ & $-0,0309$ \\
0,3 & $-0,0005$ & $-5,4287$ & $-3,2327$ & $-0,1394$ & $-0,3048$ & $-0,0826$ \\
0,4 & $-0,0010$ & $-9,4044$ & $-5,7762$ & $-3,0365$ & $-0,9048$ & $-0,1782$ \\
0,8 & $-0,0024$ & $-7,2739$ & $-10,7403$ & $-39,5282$ & $-9,6623$ & $-1,4354$
\end{tabular}

Table 9: Relative error in determination of MPC criterion using approximate eigen-modes.

Results of numerical evaluation of $\triangle \mathrm{MPC}$ are obvious from Table 9. The range of the method applicability referring $\zeta_{d}$ has been confirmed once again. Another parameter worthy to be evaluated can be defined according to paper [28] as follows:

$$
\begin{aligned}
& \operatorname{MACX}\left(v_{j, g(e x)}, v_{j, g(a p p)}\right)= \\
& =\frac{\left(\left|v_{j, g(e x)}^{*} \cdot v_{j, g(a p p)}\right|+\left|v_{j, g(e x)}^{T} \cdot v_{j, g(a p p)}\right|\right)^{2}}{\left(v_{j, g(e x)}^{*} \cdot v_{j, g(e x)}+\left|v_{j, g(e x)}^{T} \cdot v_{j, g(e x)}\right|\right)\left(v_{j, g(a p p)}^{*} \cdot v_{j, g(a p p)}+\left|v_{j, g(a p p)}^{T} \cdot v_{j, g(a p p)}\right|\right)} .
\end{aligned}
$$

The argument $x$ of eigen-modes $v_{j, g}(x)$ has been omitted due to excessive equation length. The asterisk * in Eq. (39) means the complex conjugate value. The MACX parameter is equal unity for identical eigen-modes and zero for absolutely different eigen-modes. The reverse values of MACX parameter, which compare the exact and approximate solution of complex eigen-modes, are given in Table 10. The MACX criterion showed very good accuracy in determination of eigen-modes although the phase shift differences are for some $\zeta_{d}$ significant, see e.g. Figure 4. Therefore MACX criterion could be recommended as a tool for a general identification and pairing of corresponding eigen-modes obtained from a couple of experiments rather than for the detailed analysis of differences between them.

\begin{tabular}{ccccccc}
\hline$\zeta_{d}$ & \multicolumn{6}{c}{$1-\operatorname{MACX}\left(v_{j, g(e x)}, v_{j, g(\text { app })}\right)$} \\
\cline { 2 - 7 }$[/]$ & $\mathrm{j}=1$ & 2 & 3 & 4 & 5 & 6 \\
\cline { 2 - 7 } 0 & $9 \mathrm{e}-14$ & $4 \mathrm{e}-10$ & $3 \mathrm{e}-10$ & $3 \mathrm{e}-11$ & $8 \mathrm{e}-12$ & $7 \mathrm{e}-12$ \\
0,1 & $4 \mathrm{e}-10$ & $4 \mathrm{e}-5$ & $2 \mathrm{e}-5$ & $7 \mathrm{e}-6$ & $2 \mathrm{e}-6$ & $2 \mathrm{e}-7$ \\
0,2 & $8 \mathrm{e}-9$ & 0,0004 & 0,0004 & 0,0001 & $4 \mathrm{e}-5$ & $4 \mathrm{e}-6$ \\
0,3 & $5 \mathrm{e}-8$ & 0,0007 & 0,0014 & 0,0010 & 0,0002 & $2 \mathrm{e}-5$ \\
0,4 & $2 \mathrm{e}-7$ & 0,0002 & 0,0038 & 0,0037 & 0,0006 & 0,0001 \\
0,8 & $7 \mathrm{e}-6$ & 0,0120 & 0,0479 & 0,0008 & 0,0037 & 0,0008
\end{tabular}

Table 10: Reverse values of MACX criterion of corresponding complex eigen-modes as functions of $\zeta_{d}$. 


\section{CONCLUSIONS}

Approximate analysis of modal characteristics of one dimensional non-proportionally damped system with continuously distributed mass, stiffness and damping has been outlined. The basic structure is considered proportionally damped. Subsequently attached parts, e.g. vibration absorber, with strongly different damping are sources of the non-proportional damping of the system as a whole. The core of the study consists in extension of applicability of modal characteristics acquired for a system with proportional or zero damping into a system non-proportionally damped. As a basic tool the first order perturbation procedure has been applied.

It can be concluded that the proposed procedure is flexible and easily applicable for practical purposes. It enables wide choice of generalized coordinates when keeping their character of eigen-modes of the similar operator. Concerning final eigen-modes, the structure of results is very transparent with a possibility of detailed analysis of individual components. The approach used enabled to show an equivalence of the eigen-value of the proportionally and relevant nonproportionally damped systems. On the other hand certain limits of the approach developed should be respected. In particular the system eigen-frequencies should be well separated in order to ensure a good convergence increasing number of basis functions being taken into account. Only simple eigen-values are admitted and so effect of double (or multiple) frequency splitting cannot be analyzed. The damping itself should be small and far enough from the critical damping.

Wide numerical experiments of the model of existing slender structure equipped with absorber have been carried out investigating interaction of eigen-modes between resonance domains. Numerical experiments approved a good convergence on the level of eigen-modes of the operator with continuously distributed parameters as well as on the level of eigen-modes transforming the problem to the system of generalized coordinates. First six undamped eigenmodes being close to eigen-value of the absorber revealed to be satisfactory as Galerkin base for reduction of the original problem without any impact on the accuracy of all subsequently obtained results.

The accuracy of the perturbation method applied for estimation of complex modal properties has been investigated for several damping ratios of the absorber. The comparison of approximate and exact solutions has showed increasing errors in eigen-values with an increase in damping ratio of the absorber. The error of the real part representing the "frequency" component of an eigen-value was substantially lower than that of the imaginary part representing the eigen-value "damping" component. The significant errors came up for eigen-values which were close to eigen-value of the absorber. Similarly to eigen-values, the accuracy of approximate solution of complex eigen-modes dropped with increasing absorber damping ratio as well. The complex eigen-modes have been for very low damping ratio of the absorber almost identical with real undamped eigen-modes and the mechanical interaction has been negligible. For higher values of ratio the interaction of eigen-modes, especially those that are very close in their eigen-frequencies to the tuning frequency of the absorber, has been substantial. Hence there exist a certain limit of the damping ratio, which leads to the highest phase shift variability within the eigen-modes, beyond of which the complexity of the eigen-modes is dropping although the interaction of those is increasing.

The comparison with the exact eigen-solution reveals that the approximate perturbation strategy is entitled to assess dynamic behavior of existing structures provided with one passive damping element for realistic damping ratio. In our case a limiting value of the damping ratio of the absorber for which the errors were reasonable was equal $30 \%$. Beyond this value the 
errors could be significant and more sophisticated approach should be employed.

\section{ACKNOWLEDGEMENT}

The kind support of the Czech Science Foundation project No. 103/09/0094 and No. 1341574P, Grant Agency of the ASCR project No. IAA200710902 and of the RVO 68378297 institutional support are gratefully acknowledged.

\section{REFERENCES}

[1] J. Náprstek, S. Pospíšil, S. Hračov, Random response of one-dimensional structure with non-proportional damping due to wind excitation. I. Zolotarev et al. eds. Proc. Engineering Mechanics 2007 , Svratka, Czech Republic, 2007.

[2] S. Hračov, J. Náprstek, S. Pospíšil, Non-proportional damping effect on dynamic response of footbridges. J.Náprstek, C.Fischer eds. Proc. Engineering Mechanics 2009, Svratka, Czech Republic, 2009.

[3] W. C. Hurty, M. F. Rubinstein, Dynamics of Structures. Prentice-Hall,Inc., 1964.

[4] A. S. Veletsos, C. E. Ventura, Modal analysis of non-classically damped linear systems Earthquake Engineering and Structural Dynamics. 14, 217-243, 1986.

[5] I.W. Park, J.S. Kim, F. Ma, Characteristics of modal coupling in non-classically damped systems under harmonic excitation. Journal of Applied Mechanics, 61, 77-83, 1994.

[6] H. N. Özgüven, Twenty Years of Computational Methods for Harmonic Response Analysis of Non-Proportionally Damped Systems. Proceedings of the 20th International Modal Analysis Conference, Los Angeles, CA, USA, 2002.

[7] D. E. Newland, Mechanical Vibration Analysis and Computation. Longman, Harlow and John Wiley, New York, 1989.

[8] G. B. Warburton, S. R. Soni, Errors in response calculations for non-classically damped structures. Earthquake Engineering and Structural Dynamics, 5, 365-376, 1977.

[9] G. Prater Jr., R. Singh, Quantification of the extent of non-proportional viscous damping in discrete vibratory systems. Journal of Sound and Vibration, 104(1), 109-125, 1986.

[10] W. Gawronski, J.T. Sawicki, Response errors of non-proportionally lightly damped structures. Journal of Sound and Vibration, 200(4), 543-550, 1997.

[11] A. Ibrahimbegovic, E. L. Wilson, Simple numerical algorithms for the mode superposition analysis of linear structural systems with non-proportional damping. Computer and Structures, 33 (2), 523-531, 1989.

[12] F. E. Udwadia, R. Kumar, Iterative methods for non-classically damped dynamic system. Earthquake Eng. And Structural Dynamics, 23 , 137-159,1994.

[13] S. Adhikari, Rates of change of eigenvalues and eigenvectors in damped dynamic system. AIAA Journal, 39(11), 1452-1457, 1999. 
[14] J. Woodhouse, Linear damping models for structural vibration, Journal of Sound and Vibration, 215 (3), 547-569, 1998.

[15] S. Adhikari, Calculation of derivative of complex modes modes using classical normal modes. Computers \& Structures, 77, 625-633, 2000.

[16] S. Hračov, J. Náprstek, S. Pospíšil, Analysis of eigen-values of viscously damped two degrees of freedom system due to changes of parameters of secondary subsystem. F. Soukup et al. eds. Proc. Dynamics of Rigid and deformable bodies 2011, Ústí nad Labem, Czech Republic, 2011.

[17] S. D. Garvey, J. E. T. Penny, M. I. Friswell, The relationship between the real and imaginary parts of complex modes. Journal of Sound and Vibration, 212 (1), 75-83, 1995.

[18] C. Rajakumar, Lanczos Algorithm for the Quadratic Eigenvalue Problem in Engineering Applications. International Jornal of Numerical Methods in Engineering, 105, 1-22, 1993.

[19] P. Fischer, Eigensolution of nonclassically damped structures by complex subspace iteration. Computer Methods in Applied Mechanics and Engineering, 189 (1), 149-166, 2000.

[20] J.A. Main, S. Krenk, Efficiency and tuning of viscous dampers on discrete systems. Journal of Sound and Vibration, 286, 97-122, 2005.

[21] F. Perotti, Analytical and numerical techniques for the dynamic analysis of non-classically damped linear systems. Soil Dynamics and Earthquake Engineering, 13, 197-212, 1994.

[22] J. Tang, W. L. Wang, Perturbation method for determining eigensolutions of weakly damped systems. Journal of Sound and Vibration, 187 (4), 671-681, 1995.

[23] P. D. Cha, Approximate eigensolutions for arbitrarily damped nearly proportional systems. Journal of Sound and Vibration, 288, 813-827, 2005.

[24] Y. Zhou, On the eigenvalues of specially low-rank perturbed matrices. Applied Mathematics and Computation, 217, 10267-10270, 2011.

[25] V. Koloušek, M. Pirner, O. Fischer, J. Náprstek, Wind Effects on Civil Engineering Structures. Academia-Elsevier, Prague, 1983.

[26] S.G. Michlin, Variational Methods in Mathematical Physics (in Russian). GITTL, Moscow, 1975.

[27] R. S. Pappa, K. B. Elliot, A. Schenk, Consistent-mode indicator for the eigensystem realization algorithm. Journal of Guidance, Control and Dynamics, 16(5), 852-858, 1993.

[28] P. Vacher, B. Jacquier, A. Bucharles, Extensions of the MAC Criterion to Complex Modes. Proceedings of ISMA International Conference on Noise and Vibration Engineering, Leuven, Belgium, 2010. 